\title{
Prevalence of Amblyogenic Risk Factors in Congenital Nasolacrimal Duct Obstruction in a Developing Country
}

\author{
Usha Kaul Raina, Shruti Bhattacharya*, Shantanu Kumar Gupta, Varun Saini, Banu Pavitra, \\ Kumar Ravinesh, Russell Da Cruz
}

Department of Ophthalmology, Guru Nanak Eye Centre, Maulana Azad Medical College, New Delhi, India

Email address:

shrutibhattacharya1993@gmail.com (S. Bhattacharya)

*Corresponding author

\section{To cite this article:}

Usha Kaul Raina, Shruti Bhattacharya, Shantanu Kumar Gupta, Varun Saini, Banu Pavitra, Kumar Ravinesh, Russell daCruz. Prevalence of Amblyogenic Risk Factors in Congenital Nasolacrimal Duct Obstruction in a Developing Country. International Journal of Ophthalmology \& Visual Science. Vol. 6, No. 3, 2021, pp. 176-180. doi: 10.11648/j.ijovs.20210603.15

Received: August 2, 2021; Accepted: August 23, 2021; Published: September 3, 2021

\begin{abstract}
PURPOSE: Conventionally, congenital nasolacrimal duct obstruction (NLDO) has been treated as an entity with no bearing on development of visual acuity and routine refraction is not done considering the volume of patients in a in a developing Asian country like India. This research was designed to study the prevalence of amblyogenic refractive error in patients with congenital NLDO, as compared to an age-matched control group, in a developing country. METHODS: In this study, 89 patients with congenital NLDO and 78 patients as age matched controls, less than 4 years of age were included at a tertiary care centre in India. All underwent a complete ocular examination and risk factors for amblyogenic refractive error were noted based on the AAPOS guidelines. RESULTS: There was no gender predisposition and no significant difference between the two groups in terms of mode of delivery, orthoptic check-up, keratometry, axial length, fundus or anterior segment examination. Amblyogenic refractive error, as defined by the AAPOS guidelines, was found in 18 (20.5\%) patients in the case group compared to $3(3.8 \%)$ in the control group, and this was as follows: astigmatism in 10, anisometropia in 5 , hyperopia in 3 subjects, and myopia in 1 . These risk factors were not greater in children with unilateral NLDO compared to bilateral NLDO, but both were greater than their control group. There was also no significant difference found between the two groups in terms of distribution of anisometropia. CONCLUSIONS: The prevalence of amblyogenic refractive error in congenital NLDO was significantly higher than in the control group. Hence, a thorough evaluation is warranted in cases of congenital NLDO for early detection amblyogenic refractive error.
\end{abstract}

Keywords: Nasolacrimal Duct Obstruction, Refraction, Amblyopia

\section{Introduction}

In India, infants often present with complaints of recurrent watering and intermittent discharge from one or both eyes since their birth, and are commonly diagnosed as congenital nasolacrimal duct obstruction (NLDO), with incidence of symptoms ranging from $1 \%$ to $30 \%[1,2]$ This condition is generally treated as a benign one, as most resolve spontaneously [3]. And intervention, in the form of lacrimal probing, is undertaken only after a year of no resolution [4].

Conventionally, NLDO has been treated as an entity with no bearing on development of visual acuity and routine refraction is not done considering the volume of patients in a in a developing Asian country like India.

Normal development of the visual system in early life requires the presence of a sharply focused retinal image. So hypothetically, persistent watering in congenital NLDO can lead to blurring of vision and form-deprivation amblyopia during the sensitive period of visual development [5].

Amblyopia is known to affect $1.6 \%-3.6 \%$ [6] of the general population. Previous studies [7-21] have shown a positive association between infants suffering from congenital NLDO and the presence of amblyogenic factors in them. If such an association truly exists, it would suggest a reform in our pre-existing protocol in India, with infants and children who present with congenital NLDO being screened 
for risk factors for subsequent amblyopia by fundus examination and refraction, in order to detect these risk factors, and prevent and treat amblyopia in them as soon as possible.

\section{Patients and Methods}

Approval for the study was obtained from the institutional review board of the teaching hospital. A prospective and comparative study was performed for a period of one year on patients below 4 years presenting to our tertiary centre, with complaints of epiphora, and who were diagnosed with NLDO. Inclusion criteria included epiphora, discharge from birth which did not respond to nasolacrimal duct massage or if the child had a previously confirmed diagnosis of congenital NLDO since birth, they were included in the study upto the age of 4 years. Exclusion criteria consisted of any ocular pathology which could influence refractive status, for instance media opacities, like corneal opacities or cataracts, glaucoma or children who have a prior history of surgery.

Written informed consent was obtained from the parents. Age matched controls were taken from presenting to the outpatient department with no complaints suggestive of NLDO.

Data on the following was taken: detailed history, patients' gestational age at birth, age at presentation, mode of delivery, if the NLDO was unilateral or bilateral, if right eye or left eye was involved or presence of any associated systemic diseases. Eye examinations was performed by the same observer consisting of orthoptic assessment; complete anterior segment evaluation; refraction under $1 \%$ atropine, with its sphere/cylinder/axis and its spherical equivalent; keratometry with RIGHTON - Retinomax 3 handheld Refract-Keratometer; axial length using PAC SCAN 300Ap A scan Biometer, and dilated fundus examination.

Follow up lacrimal probing under general anesthesia was done at the age of one year in cases of NLDO not resolving with conservative treatment. In the case of children who presented to us between 1 to 2.5 years of age, a trial of conservative therapy (lacrimal massage) was given for 2 months, following which they were taken up for lacrimal probing. Children older than this were given conservative treatment, and counselled for a pediatric dacryocystorhinostomy (DCR) operation later.

Risk factors for amblyopia were noted based on the American Association for Pediatric Ophthalmology and Strabismus (AAPOS) [1] guidelines. The following were considered as risk factors: anisometropia greater than $1.5 \mathrm{D}$, hyperopia greater than $3.5 \mathrm{D}$ in any meridian, myopia magnitude greater than $3.0 \mathrm{D}$ in any meridian, or astigmatism greater than $1.5 \mathrm{D}$ at $90^{\circ} / 180^{\circ}$ or greater than $1.0 \mathrm{D}$ in oblique axis.

The variables were compared between the two groups using the Chi Square, Wilcoxon and Fisher test, while those within the group with respect to various quantitative variables compared using the Chi square test, Fisher's exact test, Wilcoxon test, as per the parameters. Statistical package for Social Sciences (SPSS) was used for statistical analysis.

\section{Results}

A total of 167 children were studied, out of which 89 children were diagnosed as NLDO and 78 children were taken as age matched controls.

The median age at presentation was $13.81 \pm 10.12$ months in cases \& $17.26 \pm 12.41$ months in controls $(\mathrm{p}=0.08)$. There was no gender predisposition - the case group had $49.4 \%$ males while $57 \%$ of the control group were males.

No predisposition to laterality was noted in the case group. $56.2 \%$ were unilateral, out of which $27(30.3 \%)$ involved the right eye, and $23(25.8 \%)$ involved the left eye,

In the case group, $41(46.1 \%)$ children were full term normal vaginal deliveries and $48(53.9 \%)$ were born via a caesarian section, while in the control group, 52 (66.7\%) were full term normal vaginal deliveries and the rest were born via a cesarean section.

Anterior segment examination, fundus and orthoptic check-up was done by the same observer and were found to be normal in all.

The parameters compared between the case and control group include average keratometry $(\mathrm{K})$ of the right and left eye, and axial length (AL) of the right and left eye (Table 1).

All the 89 cases were managed conservatively with Crigler's massage and four times daily topical antibiotics. 16 out of these did not resolve, and they were taken up for lacrimal probing.

All children underwent refraction under 1\% atropine. In the case group, $18(20.5 \%)$ of the patients had high risk for amblyopia because of significant refractive error as per the AAPOS guidelines, as compared to $3(3.8 \%)$ in the control group (Table 2). All these children were prescribed glasses, and kept on a close follow up for monitoring any signs of amblyopia.

Amongst those with high risk factors, the most common refractive error as far as potential risk for amblyopia is concerned, was astigmatism (10), anisometropia (5), hyperopia (3), and myopia (1) - (Table 3).

As discussed above, $5(5.7 \%)$ children in our case group had anisometropia while $2(2.6 \%)$ in our control group had anisometropia. Hence, there appears to be no significant difference between the two groups in terms of distribution of anisometropia but this association needs a larger sample size for a definite comment.

$56.2 \%$ of the children in the case group had unilateral NLDO while the rest were bilateral. However, we did not find a higher risk of amblyopia in unilateral cases, as compared to the bilateral group. In the 9 children with unilateral disease who had refractive error with amblyogenic potential, 4 had anisometropia, 2 had astigmatism, 1 had myopia and 2 had hyperopia. 
Table 1. Comparision of Keratometry and Axial Length.

\begin{tabular}{llll}
\hline Parameters & Group: Case $(\mathbf{n}=\mathbf{8 9})$ & Group: Control $(\mathbf{n}=\mathbf{7 8})$ & p value \\
\hline Average K (Right) & $7.60 \pm 0.32$ & $7.49 \pm 0.17$ & 0.009 \\
Average K (Left) & $7.61 \pm 0.32$ & $7.50 \pm 0.19$ & 0.035 \\
Axial Length (Right) & $20.18 \pm 1.21$ & $20.07 \pm 1.68$ & 0.825 \\
Axial Length (Left) & $20.15 \pm 1.21$ & $20.09 \pm 1.70$ & 0.921 \\
\hline
\end{tabular}

Table 2. Association Between Group and High Risk for Amblyopia.

\begin{tabular}{|c|c|c|c|c|c|}
\hline \multirow{2}{*}{ High Risk for Amblyopia } & \multicolumn{3}{|l|}{ Group } & \multicolumn{2}{|c|}{ Chi-Squared Test } \\
\hline & Case & Control & Total & $\mathrm{X}^{\wedge} \mathbf{2}$ & P Value \\
\hline Present & $18(20.5 \%)$ & $3(3.8 \%)$ & $21(12.7 \%)$ & & \\
\hline Absent & $70(79.5 \%)$ & $75(96.2 \%)$ & $145(87.3 \%)$ & 10.322 & 0.001 \\
\hline Total & $88(100.0 \%)$ & $78(100.0 \%)$ & $166(100.0 \%)$ & & \\
\hline
\end{tabular}

Table 3. Association Between Group and High Refractive error.

\begin{tabular}{llll}
\hline Parameters & Group: Case $(\mathbf{n}=\mathbf{8 9})$ & Group: Control $(\mathbf{n}=\mathbf{7 8})$ & p value \\
\hline Hyperopia $>3.5 \mathrm{D}$ & $3(3.4 \%)$ & $0(0.0 \%)$ & 0.2493 \\
Myopia $>$ 3D & $1(1.1 \%)$ & $0(0.0 \%)$ & 1.0003 \\
High Astigmatism & $11(12.4 \%)$ & $2(2.6 \%)$ & 0.0181 \\
Anisometropia & $4(4.5 \%)$ & $2(2.6 \%)$ & 0.6863 \\
\hline
\end{tabular}

\section{Discussion}

The current study found a higher prevalence of amblyogenic refractive error in patients with congenital NLDO as compared to the age matched control group. However, literature is divided on the association of amblyopia risk factors with congenital NLDO.

Several studies have found a positive link between the two. Chalmers and Griffiths [7] found a 3.8\% prevalence of of anisometropic amblyopia among 130 cases of congenital NLDO, with severe hyperopia occurring in the same eye with epiphora. Matta et al [14] also identified amblyopia risk factors in 88 children $(22 \%)$ of 402 patients with congenital NLDO. This included amblyogenic refractive error in 65 (74\%), strabismus in $9(10 \%)$, and a combination of two in $14(16 \%)$. In addition, they reported [8] that the percentage of children with congenital NLDO identified retrospectively with amblyopia risk factors who later develop clinical amblyopia was much higher than the $1.6 \%$ to $3.6 \%$ expected in a cohort of normal children.

Additionally, Lacey et al [15] also found the incidence of amblyopia in a children who had undergone NLD probing to be high - $10 \%$ - as compared to an average of $2-4 \%$ in the general population.

There are only three major Asian studies on this topic.

Kim et al [18], found a prevalence of $35 \%$ prevalence of amblyopia risk factors in NLDO in Asian children, which is much higher than the average. However, they had a very small sample size of just 25 patients.

Badakere et al [16] also found the prevalence of amblyopia risk factors in children with unilateral congenital NLDO to be marginally higher than that reported in general population, with $14 \%$ of their patients having amblyopic risk factors, while Ramkumar et al [17] again reported similar rates of prevalence of amblyopia risk factors in congenital NLDO (14\%).
Some trials, however, have found no such correlation.

Ellis et al [19] reviewed a cohort of 4792 children with congenital NLDO and found no evidence of an adverse effect of congenital NLDO on visual development, when compared to controls, nor in the watering eye compared to the fellow eye.

Yoo et al [20] found no increased prevalence of amblyogenic refractive errors in such children, and with no difference in prevalence between unilateral and bilateral NLDO.

In a recent study, Vagge et al [21] found no association between the two - they reported the prevalence of amblyopia risk factors to be $11.9 \%$ in infants aged 30 to 60 days with NLDO versus $8.7 \%$ in age matched control subjects.

Along with these contended results about amblyopia in general in these children, anisometropia in association with unilateral NLDO, has also been reported found as a risk factor in several studies. In our study, we found no such association, with the prevalence rates for amblyogenic risk factors being similar in both unilateral and bilateral cases.

Similar results have been found in other studies as well. Ramkumar et al [17] also found no correlation between the laterality of congenital NLDO and the degree of refractive error in children, and Nair et al [22], again found no difference in the development of amblyopia in unilateral versus bilateral disease.

Also, in the study by Vagge et al [21], no difference in amblyopia risk factors was found in eyes with unilateral $(11.5 \%)$ and bilateral $(12.1 \%)$ congenital NLDO, or in eyes with unilateral congenital NLDO and fellow eyes $(9.8 \%$ versus $12.3 \%$ ).

Some studies, however, have found a positive correlation between anisometropia and amblyopia. Badakere et al $\left[16^{]}\right.$ noted anisometropia in five patients and showed the worse eye to be the one with congenital NLDO in all the cases. Saleem et al [9] also found unilateral congenital NLDO to be associated with statistically significant anisometropia.

Simon et al [10] reported 5 cases of congenital NLDO with 
anisometropic amblyopia developing on the same side, with all these 5 patients having hyperopic anisometropia in the eye with NLDO.

Kipp et al [11] observed that anisometropia occurred at a greater rates in unilateral NLDO patients $(7.6 \%)$ compared with bilateral NLDO (3.6\%), with $5.8 \%$ of the patients developing clinical amblyopia - with the rate of development into amblyopia increasing to $22 \%$ for anisometropics.

Pelit et al [23] found that anisometropia more than 0.5 dioptres (D) was present in $11.7 \%$ of patients with unilateral NLDO, and hyperopia was common in the affected eyes.

Finally, Piotrowski et al [13], after reviewing data from 305 congenital NLDO patients, concluded that children with congenital NLDO develop anisometropia with or without amblyopia more frequently than children in the general population.

\section{Strengths and Limitations of Our Study}

With many such contentious studies, the strengths of our study include uniform protocols, inclusion of keratometry and axial length in the ocular investigations, examination by the same ophthalmologist and presence of an age matched control group for comparison.

However, our study had many limitations: a relatively small study size, exclusion of patients with strabismus or congenital cataract, and lack of long term follow up data on whether those patients with high risk for amblyopia actually developed amblyopia later on in life.

\section{Conclusion}

We did find a significantly higher prevalence of amblyogenic risk factors in children with NLDO, and we may infer that the current research supports a correlation between NLDO and amblyopia, but we need large multicentric trials with a control group for definite results.

On the basis of the conclusions of our study, we recommend that along with regular management of NLDO, special attention also needs to be paid to a through ophthalmological examination in children presenting with congenital NLDO to us in India, with orthoptics, retinoscopy and a close follow up, in order to prevent the dire consequence of amblyopia in them.

\section{Institution}

Study conducted at Pediatric Ophthalmology Services, Guru Nanak Eye Centre, New Delhi, India - 110002.

\section{Conflict of Interest}

No conflicting relationship exists for any author.

Registration number - CTRI/2019/03/018057 in Clinical Trials Registry - India (ICMR-NIMS).

\section{Statement of Equal Authors' Contribution}

All the authors meet the requirements for authorship. The manuscript has been read by all the authors and each author believes that the manuscript represents honest work. There has been no prior publication of this work and none of the authors have any financial interest in any of the materials and methods used in this study. I would like to mention that the manuscript, to the best of the author's knowledge, does not infringe upon any copyright or property right of any third party.

\section{Declarations}

1. Ethics approval - yes, from the institutional review board of the teaching hospital

2. Consent to participate - yes

3. Consent for publication - yes

\section{Abbreviations}

AAPOS: American Association For Pediatric Ophthalmology And Strabismus

NLDO: Nasolacrimal Duct Obstruction

\section{References}

[1] Donahue SP, Arnold RW, Ruben JB, AAPOS Vision Screening Committee. Preschool vision screening: what should we be detecting and how should we report it? Uniform guidelines for reporting results of preschool vision screening studies. J AAPOS Off Publ Am Assoc Pediatr Ophthalmol Strabismus. 2003; 7 (5): 314-316.

[2] Macewen CJ, Young JDH. Epiphora during the first year of life. Eye. 1991; 5 (5): 596-600.

[3] Kakizaki H. The rate of symptomatic improvement of congenital nasolacrimal duct obstruction in Japanese infants treated with conservative management during the 1 st year of age. Clin Ophthalmol. 2008; 2 (2): 291.

[4] Vagge A, Ferro Desideri L, Nucci P, et al. Congenital Nasolacrimal Duct Obstruction (CNLDO): A Review. Diseases. 2018; 6 (4): 96.

[5] Aslam Saleem A. Congenital Nasolacrimal Duct Obstruction and the Visual System. Front Ophthalmol Ocul Imaging. June 2019.

[6] Friedman DS, Repka MX, Katz J et al. Prevalence of amblyopia and strabismus in white and African American children aged 6 through 71 months the Baltimore Pediatric Eye Disease Study. Ophthalmology. 2009; 116 (11): 2128-34.

[7] Chalmers R GP. Is congenital nasolacrimal duct obstruction a risk factor for the development of amblyopia. Br Orthop $J$. 1996; (53): 29-30.

[8] Matta NS, Silbert DI. High prevalence of amblyopia risk factors in preverbal children with nasolacrimal duct obstruction. J AAPOS. 2011; 15 (4): 350-352. 
[9] Saleem AA, Siddiqui SN, Wakeel U, Asif M. Anisometropia and refractive status in children with unilateral congenital nasolacrimal duct obstruction. Taiwan J Ophthalmol. 2018; 8 (1): 31-35.

[10] Simon JW, Ngo Y, Ahn E, Khachikian S. Anisometropic amblyopia and nasolacrimal duct obstruction. J Pediatr Ophthalmol Strabismus. 46 (3): 182-183.

[11] Kipp MA, Kipp MA, Struthers W. Anisometropia and amblyopia in nasolacrimal duct obstruction. J AAPOS. 2013; 17 (3): 235-238.

[12] Aysel Pelit NS glu-, Keskek HC. Refractive status of children with unilateral congenital nasolacrimal duct obstruction. $J$ AAPOS. 2018; 22 (4): 83.

[13] Piotrowski JT, Diehl NN, Mohney BG. Neonatal Dacryostenosis as a Risk Factor for Anisometropia. Arch Ophthalmol. 2010; 128 (9): 1166.

[14] Matta NS, Singman EL, Silbert DI. Prevalence of amblyopia risk factors in congenital nasolacrimal duct obstruction. $\mathrm{J} \mathrm{Am}$ Assoc Pediatr Ophthalmol Strabismus. 2010; 14 (5): 386-388.

[15] Lacey BA, McGinnity GF, Johnston PB, Archer DB. Congenital epiphora as a potential cause of amblyopia. Vision Research. 1995 (35): S130.

[16] Badakere A, Veeravalli Tabitha N, Iram S, Naik M, Ali MJ. Unilateral congenital nasolacrimal duct obstruction and amblyopia risk factors. Clin Ophthalmol. 2018; 12: 12551257.
[17] Ramkumar Va, Agarkar S, Mukherjee B. Nasolacrimal duct obstruction: Does it really increase the risk of amblyopia in children? Indian J Ophthalmol. 2016; 64 (7): 496.

[18] Kim JW, Lee H, Chang M, Park M, Lee TS, Baek S. Amblyopia Risk Factors in Infants With Congenital Nasolacrimal Duct Obstruction. J Craniofac Surg. 2013; 24 (4): $1123-1125$.

[19] Ellis JD, MacEwen CJ, Young JD. Can congenital nasolacrimal-duct obstruction interfere with visual development? A cohort case control study. $J$ Pediatr Ophthalmol Strabismus. 1998; 35 (2): 81-85.

[20] Yoo Y, Yang HK, Kim N, Choung HK, Hwang JM, Khwarg SI. Amblyopia risk factors in congenital nasolacrimal duct obstruction: A longitudinal case-control study. PLoS One. 2019; 14 (6): $\mathrm{e} 0217802$.

[21] Vagge A, Tulumello C, Pellegrini M, Di Maita M, Iester M, Traverso CE. Amblyopia Risk Factors in Newborns With Congenital Nasolacrimal Duct Obstruction. $J$ Pediatr Ophthalmol Strabismus. 2020; 57 (1): 39-43.

[22] Nair AA, Khandji J, Lustig A, Ranka M. Nasolacrimal duct obstruction and the development of anisometropia and amblyopia. J Am Assoc Pediatr Ophthalmol Strabismus. 2019; 23 (4): e46.

[23] Pelit A, Sahinoglu-Keskek N, Canan H. Refractive status of children with unilateral congenital nasolacrimal duct obstruction. J Am Assoc Pediatr Ophthalmol Strabismus. 2018; 22: e83-e84. 